\title{
Current Status of Endoscopic Vacuum Therapy in the Management of Esophageal Perforations and Post-Operative Leaks
}

\author{
Imogen Livingstone ${ }^{1 *}$, Lily Pollock ${ }^{1 *}$, Bruno Sgromo $^{2}$ and Sotiris Mastoridis ${ }^{2}$ \\ ${ }^{1}$ University of Oxford, Oxford, ${ }^{2}$ Oxford Oesophagogastric Centre, Churchill Hospital, Oxford University Hospitals NHS Foundation Trust, \\ Oxford, UK
}

Esophageal wall defects, including perforations and postoperative leaks, are associated with high morbidity and mortality and pose a significant management challenge. In light of the high morbidity of surgical management or revision, in recent years, endoscopic vacuum therapy (EVT) has emerged as a novel alternative treatment strategy. EVT involves transoral endoscopic placement of a polyurethane sponge connected to an externalized nasogastric tube to provide continuous negative pressure with the intention of promoting defect healing, facilitating cavity drainage, and ameliorating sepsis. In the last decade, EVT has become increasingly adopted in the management of a diverse spectrum of esophageal defects. Its popularity has been attributed in part to the growing body of evidence suggesting superior outcomes and defect closure rates in excess of $80 \%$. This growing body of evidence, coupled with the ongoing evolution of the technology and techniques of deployment, suggests that the utilization of EVT has become increasingly widespread. Here, we aimed to review the current status of the field, addressing the mechanism of action, indications, technique methodology, efficacy, safety, and practical considerations of EVT implementation. We also sought to highlight future directions for the use of EVT in esophageal wall defects. Clin Endosc 2021;54:787-797

Key Words: Endoscopic vacuum therapy; Esophagus; Leak; Perforation

\section{INTRODUCTION}

Transmural esophageal defects include those arising due to perforation and occurring postoperatively due to anastomotic leakage (AL). Esophageal perforation is predominantly the consequence of iatrogenic injury, which occurs during endoscopy or intraoperatively. Non-iatrogenic perforation is predominantly due to spontaneous/effort rupture, accounting for approximately $15 \%$ of all esophageal perforations. Other

\footnotetext{
Received: September 23, 2021 Revised: October 18, 2021 Accepted: October 18, 2021

Correspondence: Sotiris Mastoridis

Churchill Hospital, Oxford University Hospitals NHS Foundation Trust, Old Rd, Headington, Oxford OX3 7LE, UK

Tel: +44-300-304-7777, Fax: +44-186-522-3589, E-mail: sotiris.mastoridis@ouh. nhs.uk

ORCID: https://orcid.org/0000-0001-7689-2330

*These authors contributed equally to this study.

It is the invited review article.

(c) This is an Open Access article distributed under the terms of the Creative Commons Attribution Non-Commercial License (http://creativecommons.org/ licenses/by-nc/3.0) which permits unrestricted non-commercial use, distribution, and reproduction in any medium, provided the original work is properly cited.
}

rare and non-iatrogenic perforations include trauma and malignancy. In the United Kingdom, according to hospital episode statistic data, the 90 -day mortality was $38.8 \%$, although this was partly dependent on the interval between injury and the start of treatment. ${ }^{1,2} \mathrm{AL}$ is a life-threatening complication occurring in 5-30\% of patients following esophagectomy, with a reported mortality rate ranging from $20-50 \%$ of cases. ${ }^{3}$ Leakage of gastric contents into the thoracic cavity can result in mediastinitis, life-threatening sepsis, and multi-organ failure. Management frequently necessitates repeated interventions, prolonged hospital, and intensive care stay and is associated with substantial healthcare and societal costs.

Nevertheless, there are no strict guidelines on the management approaches to these complex and often life-threatening situations, with treatment often differing between clinicians, departments, and institutions. In recent years, interventional endoscopy has evolved as an effective alternative to surgical revision, and for the most part, is a highly morbid undertaking. This typically involves the application of self-expanding metal stents (SEMS) for defect closure and cavity exclusion coupled with external drainage. Additionally, alternative endoscopic 
approaches include clipping, endoscopic suturing, and fibrin glue injection. While each has had its proponents, reports of efficacy and safety have been highly variable. ${ }^{4}$

Since its first description in 2008, ${ }^{5}$ endoscopic vacuum therapy (EVT) has emerged as a promising alternative in the management of esophageal wall defects. The technique, which draws on the principles and experience of topical negative pressure in the management of superficial wounds, involves the transoral endoscopic placement of a polyurethane sponge connected to an external continuous negative pressure device via a nasogastric tube to promote defect healing, facilitate cavity drainage, and ameliorate sepsis.

Since its introduction, the evidence-based approach in the context of esophageal defects has grown, with over 400 reported cases in the literature and defect closure success rates described to be over $80 \%{ }^{6}$ As a consequence, the technique has gained increasing attention and popularity among gastroenterologists and surgeons. In this review, we aimed to discuss the emerging role of EVT in the management of gastrointestinal transmural defects, including its mechanisms of action, indications, methodology, efficacy, and safety. We also aimed to highlight future avenues in which this exciting technique may come to the fore.

\section{MECHANISTIC PRINCIPLES}

The mechanistic principles by which negative pressure therapy exerts therapeutic effects have been explored predominantly in the context of superficial wound healing and extended to the context of the gastrointestinal tract. Broadly, this involves the mechanical apposition of wound edges to promote healing, granulation of healthy tissue, neovascularization, control of the septic focus through active drainage, and the diversion of secretions.

Continuous negative pressure exerts mechanical deformation forces that yield macroscopic and microscopic benefits. Macrodeformation of the defect edges facilitates their contraction, closer apposition, and subsequent defect closure through the formation of granulation tissue. Microdeformation at the cytoskeletal level encourages pro-proliferative and pro-migratory cell signaling cascades and downstream expression of extracellular matrix and contractile molecules that drive defect healing. ${ }^{7}$ The vacuum forces also drive local angiogenesis through induction of transient hypoperfusion and activation of the hypoxia-inducible factor-vascular endothelial growth factor pro-angiogenic pathway, manifesting as increased blood vessel density and flow in the region. ${ }^{8}$ Such enhanced perfusion is fundamental to healing due to increased delivery of oxygen and nutrients and clearance of waste by-products. EVT may further enhance perfusion by promoting clearance of accumulated extracellular fluid and tissue edema which, taken together, serve to reduce external microvascular pressure. ${ }^{9} \mathrm{~A}$ key feature of EVT is the ability to divert salivary, gastric, and local secretions which may be irritants away from the site of healing. Furthermore, active drainage of intracavitary content serves to control septic focus. However, in instances of complex loculated cavities, simultaneous percutaneous drainage may be performed.

\section{PROCEDURAL APPROACHES}

Approaches to EVT insertion vary and reflect the range of equipment used, operator/center experience, and available resources. In addition, the nature of the defect and the patient's clinical condition must be considered. Nevertheless, all insertions and exchanges should follow certain basic principles. Initial insertion is predominantly performed in the operating room under general anesthesia with endotracheal intubation for securing the airway and minimizing aspiration risk. This facilitates a comprehensive assessment of defects and any associated cavity. On occasion, EVT exchanges can be performed in the endoscopy suite or by the bedside and, if appropriate, under sedation. Vacuum sponges have been custom-made by clinicians as previously described $;{ }^{10}$ however, most units now employ kits, among which the Eso-SPONGE ${ }^{\circledR}$ (B. Braun, Melsungen, Germany) is commonly used (Fig. 1). EsoSPONGE ${ }^{\circledR}$ insertion begins with a comprehensive endoscopic assessment, then an overtube is passed over the endoscope. Once in position, the endoscope is removed, and the sponge is advanced down the length of the overtube using the pusher. The position of the sponge can be checked endoscopically, and correction can either be achieved by gentle traction on the tubing for withdrawal or by using endoscopic graspers. Transnasal passage of the EVT tubing is then performed, and finally, continuous suction is applied using an external vacuum pump device set to a pressure of $125 \mathrm{mmHg}$ typically.

The sponge element can be placed in one of two positions relative to the defect: transmurally within the cavity itself (intracavity), or lying over the defect while remaining within the gastrointestinal tract (intraluminal). This decision is guided by several factors relating to both patient and practitioner preferences; however, key considerations include the defect size and cavity characteristics. Regular reassessment at the time of EVT exchange should be performed to confirm whether intracavity or intraluminal placement is best suited to the clinical context. In our practice, intraluminal placement is preferred whenever possible. Thus, we tend to aim for distal deployment of the sponge device at the gastroesophageal junction and then 

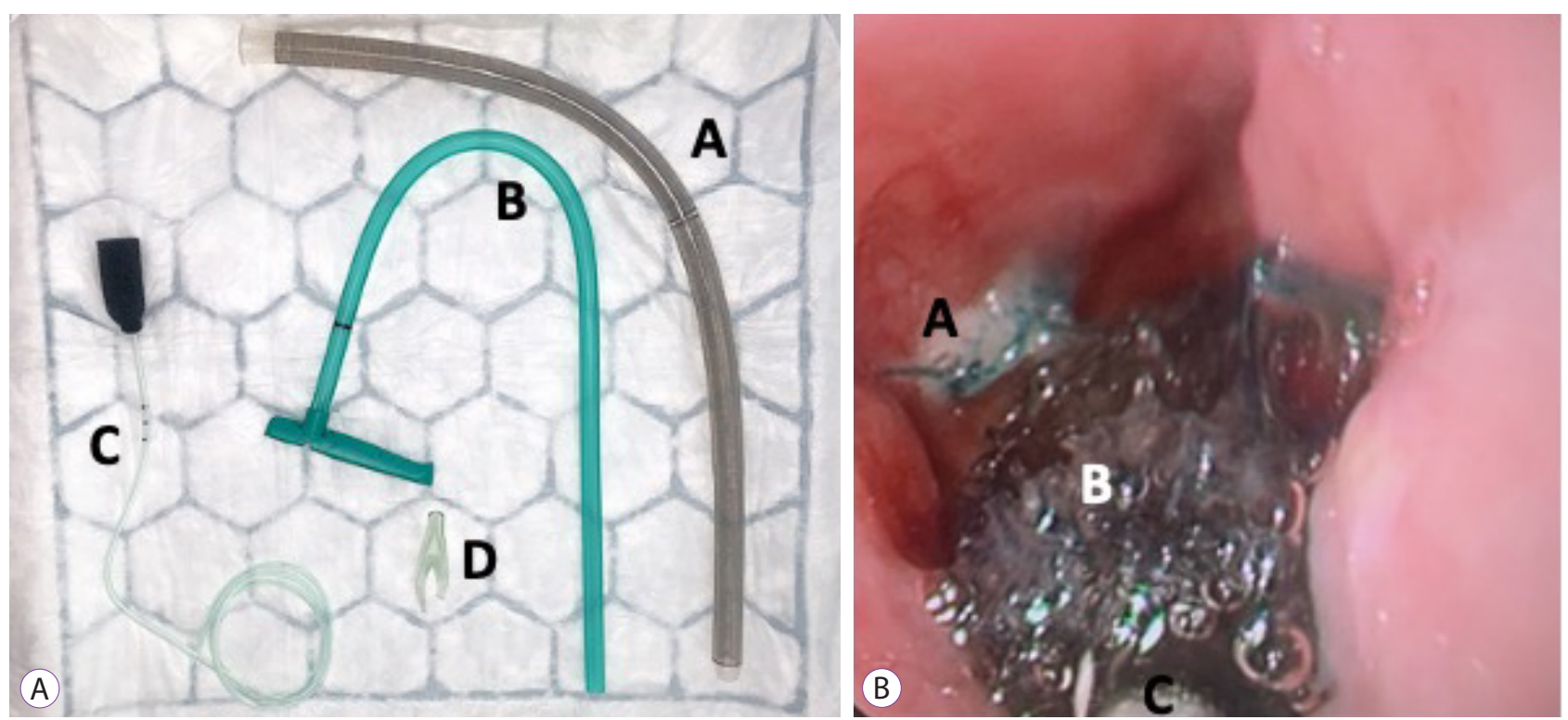

Fig. 1. (A) Eso-SPONGE ${ }^{\circledR}$ kit contents. A.Overtube with an inner diameter of $13 \mathrm{~mm}$ to allow passage of endoscope. B. Sponge pusher. C. Eso-SPONGE ${ }^{\circledR}$ unit. D. Y connecting piece. (B) Endoscopic vacuum therapy of anastomotic leak. A. Anastomotic suture line. B. Intraluminal sponge. C. Tubing.

withdraw the sponge by gentle traction on the tubing while under endoscopic imaging for the final placement overlying the pre-identified defect. Factors that preclude intraluminal placement include defects that are too large to be covered by the sponge provided in the kit. Fundamental to the efficacy of both intracavity and intraluminal EVT is the simultaneous closure of the defect and drainage of gastrointestinal secretions to provide a contaminant-free environment conducive to healing. A prerequisite for successful defect closure is the presence of a closed compartment because this allows for the establishment and maintenance of the negative pressure. Effective healing depends on adequate collapse of the wound defect under suction and intact regional perfusion. ${ }^{6}$

EVT exchange should occur between 48 and 72 hours. While many reports suggest the feasibility of longer placement periods, shorter-interval serial exchange regimens are associated with numerous advantages, such as easier removal due to less time for granulation tissue infiltration and tethering of the sponge to the lumen wall, and avoidance of loss of suction forces, which inevitably occur over time as gastrointestinal secretions exceed the drainage capacity of the foam fenestrations. When performing EVT exchange, following disconnection of the continuous negative pressure, Leeds and colleagues described a technique wherein the endoscope is used in a circumferential manner at the sponge-tissue interface to aid dislodgement from new granulation tissue, followed by gentle longitudinal traction on the tube to disengage the sponge fully back into the gastrointestinal tract lumen. ${ }^{11}$ Infusion of water or saline via the nasogastric tube can also facilitate the process. The device is then retrieved via the mouth and, finally, the tubing can be cut with scissors for the sponge to be discarded and the remaining tubing to be withdrawn from the nose.

Regular assessment should be made with regard to patient progress, the efficacy of EVT therapy, and whether ongoing therapy is appropriate. In the early period, it is important to confirm that the approach is to achieve diversion of enteric content through monitoring of drainage outputs. In the first week or two therapy, it must be determined whether effective sepsis control is achieved using clinical and biochemical parameters. Finally, successful cavitary/defect healing should be performed via regular endoscopic assessment. The above should be performed with concurrent optimization of nutritional supplementation, external drainage wherever appropriate, and concomitant antimicrobial/antifungal therapy.

\section{INDICATIONS}

Since the first reported use of EVT in the upper gastrointestinal tract in 2008, specifically in the context of intrathoracic anastomotic leak, the range and scope of utility of the approach has widened substantially to include perforations, anastomotic leaks, and fistulae in a variety of clinical settings. ${ }^{5}$ This has been demonstrated in multiple case reports across countries and institutions (Table 1), ${ }^{1,5}, 12-30$ with Germany being a particular focus of expertise and progress in the field. The 


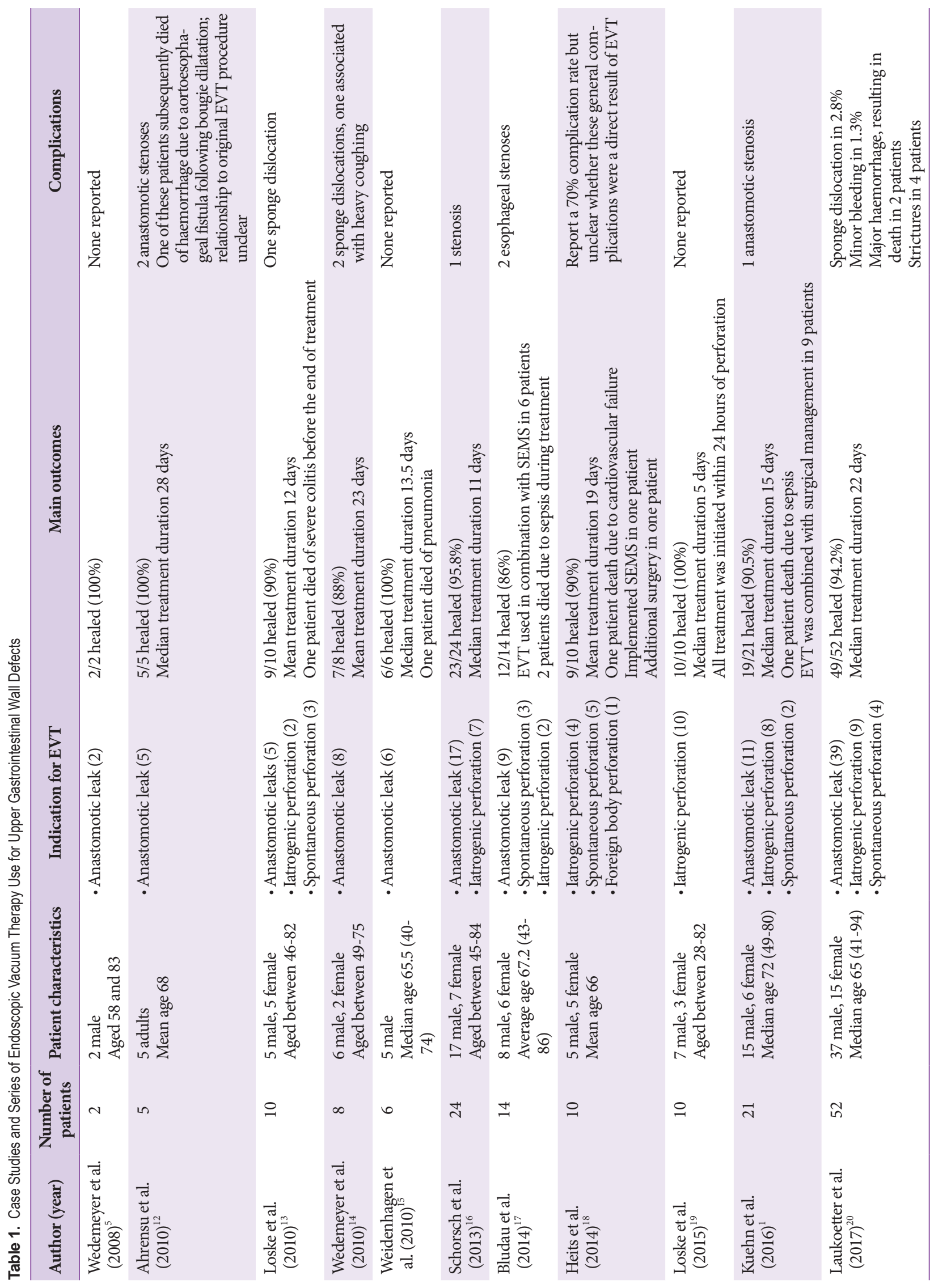




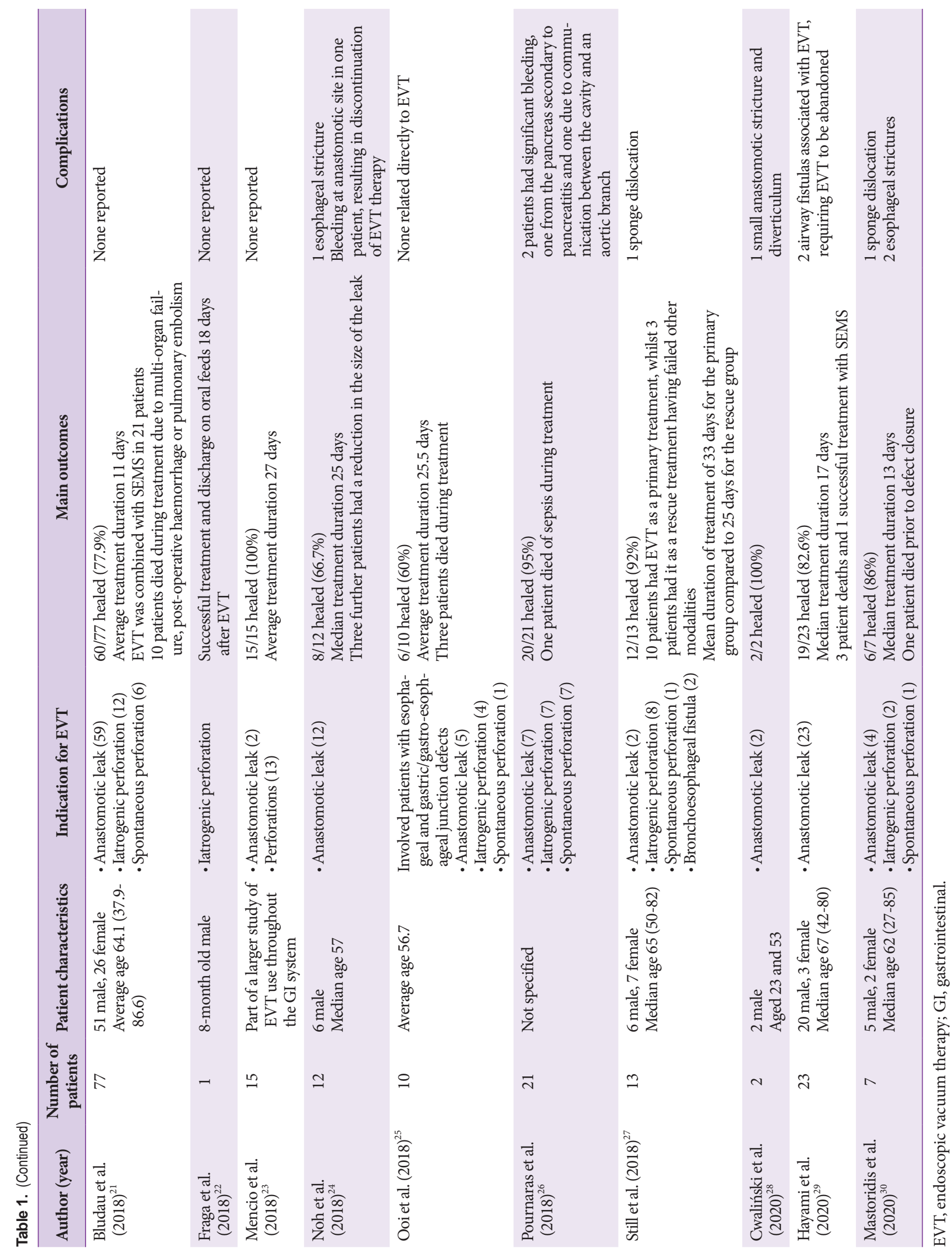


approach has been increasingly demonstrated to be efficacious across a spectrum of etiologies, ages, comorbidities, and patient conditions. Regarding age specifically, while the bulk of published evidence relates to the use of EVT in adults, there are reports of its use in pediatric cohorts. ${ }^{22,31}$ The largest of these is by Manfredi et al., ${ }^{31}$ who described 17 cases with a median age of 24 months that underwent EVT in the context of either endoscopic or surgical perforation of the esophagus. The outcomes were excellent, with successful defect closure achieved in $88 \%$ of cases. An additional benefit of EVT is that it can be used in critically ill individuals to achieve sepsis source control. In our experience, as well as those reported by others, there have been cases in which EVT was the only viable option in instances where surgical intervention was precluded by the severity of the patient's condition. The potential for EVT to serve as a temporizing measure in optimizing a patient prior to a planned definitive surgical intervention is also becoming increasingly recognized.

Although consensus on the indications for EVT therapy is yet to be established, contained and non-loculated cavities $<8$ $\mathrm{cm}$ in maximal dimension are particularly suited for intracavitary device placement. If a luminal defect is small but is associated with a contaminated cavity with the above characteristics, it may be appropriate to dilate the defect to enable intracavitary sponge positioning. Small defects that can be traversed by the length of the sponge, which either have a small associated cavity or being drained by other (e.g., percutaneous) means, can be managed by intraluminal device placement. However, there is no agreement regarding the upper limit of the defect size that can be treated intraluminally.

Although absolute and relative contraindications to EVT are not fully established, it is clear that its efficacy is limited in certain scenarios. First, there are certain defect- and cavity-related concerns that may preclude application. Among these are the presence of large food debris within the cavity, development of large multiloculated collections, and defects of greater size than can be traversed by the sponge device. While EVT could be applied in instances of complete or near-complete anastomotic dehiscence for control of sepsis, definitive surgical reintervention is required. Absolute contraindications include the proximity of visible large blood vessels, which poses a risk for hemorrhage or fistulation, and any scenario in which the continuous application of negative pressure is disrupted, such as cutaneous fistulation

It is important that prior to performing an EVT, a comprehensive assessment of a patient's pre-morbid state and treatment-related prognosis should be performed, and we recommend taking into account P-Possum (or similar) scoring. Specifically, considerations should include whether or not several procedures under anesthesia would be tolerated, and the relative merits of alternative strategies should be weighed. Multidisciplinary discussions involving surgeons, intensivists, and anesthetists, as well as the nursing staff and patient families, are central to making the best decisions regarding the appropriateness of continued EVT therapy.

\section{EFFICACY}

In our early experience at our tertiary center in Oxford, we published the largest case series of EVT in the UK and demonstrated an $86 \%$ success rate of defect closure with a median duration of treatment of 13 days. $^{30}$ Since this time, our experience has continued to grow, and we continue to observe similar rates of success. These results are consistent with previously reported EVT success rates, including the retrospective analysis of Brangewitz and colleagues, in which 34 patients demonstrated an $84 \%$ closure rate of intrathoracic esophageal leaks, ${ }^{32}$ and Bludau et al, ${ }^{17}$ who successfully closed $86 \%$ of esophageal perforations in a group of 14 patients using this technique. Another group achieved a 78\% closure rate among the largest esophageal wall defect cohort managed with EVT to date of 77 patients, which included spontaneous perforations, iatrogenic injury, and post-operative leaks. ${ }^{21}$ Others have reported higher success rates of $100 \%$ closure of esophageal leaks using $\mathrm{EVT}^{23}$ In contrast, Ooi et al. ${ }^{25}$ reported a lower success rate of $60 \%$, which was attributed to the complexity and severity of illness in their patient cohort. However, other studies involving critically ill patients and those who failed to respond to other treatment modalities revealed excellent success rates of up to $100 \%{ }^{27}$

EVT is effective not only in facilitating closure of esophageal wall defects but also in rapidly controlling sepsis by efficiently draining the associated infected wound cavities. This effect is highlighted by studies in which monitoring of inflammatory markers and patient condition have shown a significant reduction in white cell count and C-reactive protein following initiation of EVT treatment. ${ }^{12,20,25,30}$ This rapid source control helps to stabilize patients who are critically ill at the onset of EVT treatment, thus reducing the need for intensive care support, which is a major advantage of the approach. ${ }^{27}$

The timing of EVT initiation must also be considered, and prompt treatment is widely acknowledged as a major factor influencing its therapeutic outcome. In a case series focusing on acute iatrogenic endoscopic perforations, Loske et al. ${ }^{19}$ reported a success rate of $100 \%$, which is an impressive result partly attributed to the quick diagnosis of perforation and commencement of treatment within 24 hours in all cases. The treatment duration in these patients was also remarkably quick, with a median duration of 5 days and no reported com- 
plications. These findings are supported by reports of a significant difference in outcomes for patients in whom treatment was started before and after $24 \mathrm{~h}$ of defect onset. ${ }^{18}$

The efficacy of EVT has also been directly compared with the use of SEMS, a widely implemented alternative management option for esophageal wall defects. Table 2 summarizes the systematic reviews performed comparing these interventions. ${ }^{33-35}$ Overall, EVT has demonstrated superiority to SEMS for treating esophageal leaks across multiple domains, including a higher closure rate ( $84 \%$ vs. $53 \%$ ), a lower mortality rate (15\% vs. $25 \%$ ), shorter treatment duration (median 23 days vs 33 days), and a lower complication rate with regard to strictures ( $9 \%$ vs. $28 \%$ ). ${ }^{32}$ Moreover, in systemically unwell patients with post-esophagectomy anastomotic leak, EVT was reportedly associated with a much lower mortality (12\%) compared to SEMS (83\%) or surgical (50\%) management, ${ }^{36}$ highlighting its potential clinically significant benefits for patient outcomes. These results have not been replicated in all studies. Berlth et al. ${ }^{37}$ reported on a large cohort of 111 patients, in which a closure rate of $85.7 \%$ for EVT vs. $72.4 \%$ for SEMS was not found to be statistically significant $(p=0.152)$. Their study had the advantage of only including patients who were experiencing leaks after esophagectomy for cancer, whereas previous studies have included a much more heterogeneous group of patients. However, the first systematic review and meta-analysis comparing EVT to SEMS confirmed the therapeutic benefits of EVT, including a significantly higher success rate in healing esophageal leaks and perforations resulting in fewer complications and reduction in in-hospital mortality compared to SEMS. $^{34}$

EVT has also been used in combination with surgery. Kuehn et al. ${ }^{1}$ reported that 9 out of 21 patients receiving EVT treatment also underwent surgical intervention, with the authors viewing the two modalities as complementary. In these cases, esophageal wall defects that could not be closed with suturing were tackled with EVT, while concomitant mediastinitis was controlled with an established surgical approach. EVT has also been used in combination with SEMS, with the stent placed after starting EVT therapy and removed 5-6 weeks later. However, the indications for this dual treatment approach and its potential outcomes are yet to be defined..$^{17,21}$

Another important considerations are the long-term outcomes following EVT, along with patient experience. Heits et al..$^{38}$ conducted a prospective longitudinal study in a cohort of patients undergoing esophagectomy for cancer and compared the health-related quality of life outcomes (HRQOL) of patients treated with EVT for postoperative anastomotic leaks to that of patients without anastomotic leaks. Surprisingly, the results showed that patients who had undergone EVT following an anastomotic leak had better HRQOL scores than those without. Similarly, Dhavat et al. ${ }^{39}$ did a prospective survey to compare the gastrointestinal quality of life index (GIQLI) between patients treated with EVT and those treated with esophagectomy but did not experience any leak. The median GIQLI score was lower in patients who underwent EVT treatment ( 83 vs $96.5, p=0.185$ ) but comparable between groups for most domains assessed, including symptoms and physical functions. Overall, these results tentatively support EVT as a successful and acceptable approach to patients in the long term without a detrimental impact on subsequent quality of life.

\section{SAFETY}

Safety is a primary concern in the introduction of any novel technique. So far, EVT has been demonstrated to be associated with a few major complications. The greatest concern lies in the potential for major hemorrhage. Laukoetter et al ${ }^{20}$ reported on a large case series of 52 patients, in which there were two patient deaths attributed to hemorrhage associated with EVT treatment. One of these patients died following the removal of the EVT sponge during a sponge exchange procedure, which was believed to be a catastrophic rupture of the descending aorta. Ahrens et al. ${ }^{12}$ also reported a patient death caused by major bleeding after a bougie dilatation of a stricture caused by EVT therapy, where there was creation of an aortoesophageal fistula. Finally, our colleagues in Cambridge reported major bleeding in two patients; in one case, there was a direct communication of an aortic branch to the cavity, and in the other case, there was bleeding of the pancreas secondary to severe pancreatitis. Both patients were subsequently stabilized and successfully treated with EVT. ${ }^{26}$ Evidence of major bleeding during EVT may include the appearance of fresh blood in the EVT output fluid. In the case of any major bleeding, EVT should be stopped immediately, and triple-phase computed tomography (CT) is indicated to guide further management. Some have advocated for a CT scan of the thorax to be performed as a standard when initiating EVT treatment to assess the relationship of the esophageal defect to any major cardiovascular structures; hence, the risk of erosion causing major hemorrhage. ${ }^{20}$

Other reported minor adverse events include sponge dislocation, minor bleeding, and anastomotic strictures. Although not life-threatening, these complications can have a significant impact on patients; for example, anastomotic strictures have been demonstrated to have a negative and long-lasting impact on the quality of life despite treatment with endoscopic dilatation. ${ }^{38}$ It is important to note that many of these safety considerations are made on the basis of case reports and case 


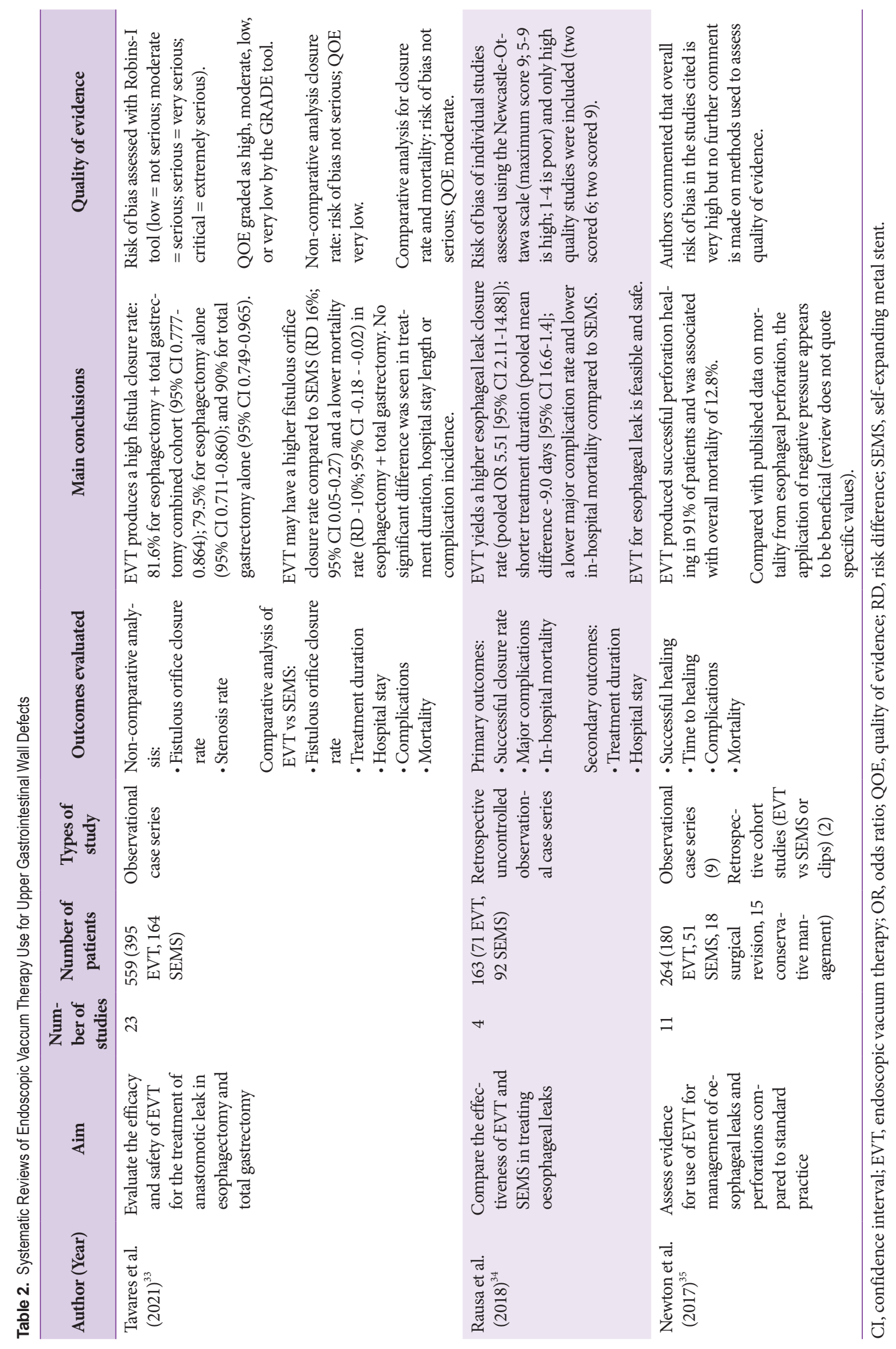




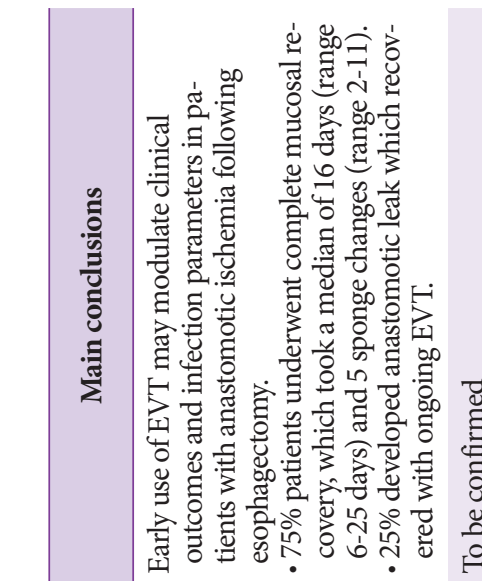

\section{चू
:
ठ
0
0
0}

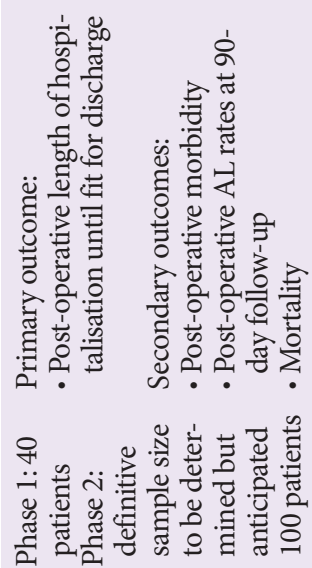

สิ่

ळे

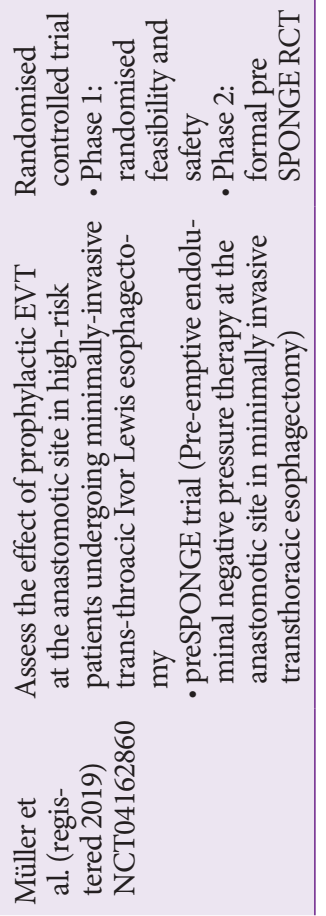

series involving limited numbers of patients. A large, multinational, prospective registry of EVT use will be crucial to gather further data and generate a greater understanding of outcomes and safety (NCT02662777).

\section{COSTS AND IMPEDIMENTS TO IMPLEMENTATION}

With the development of any new procedure in healthcare, the feasibility with which it can be widely adopted in clinical practice will inevitably be partly dependent on the cost. Cost calculations are complex; they need to be understood in relation to alternative treatment strategies, such as stenting or definitive surgical intervention. However, complexity also exists as EVT can be performed in various settings (operating room, endoscopy suite, or bedside). Moreover, its impact on the length of hospital stay, number of exchanges necessary, and the rate of complications associated with further intervention (e.g., stricture) also play a role.

These factors were considered in a study by Ooi et al. (2019), ${ }^{25}$ who estimated that the total cost of EVT was approximately $\$ 10,188(\mathfrak{E 5 , 4 1 6 )}$ per patient over a median treatment duration of 25 days. Among the eight items considered in this calculation, time in the operating room and the costs of general anesthesia were the two greatest contributors to the overall cost of the procedure. Additionally, the patients considered were critically ill; it is unlikely that costs would be equally high in patients who can be treated at the bedside and for shorter periods of time. In a retrospective analysis of 50 EVT cases, Ward et al. ${ }^{40}$ found that endoscopy suite-based procedures were associated with a 2.5 -fold lower cost than those in the operating room (with an average total cost of $\$ 4,528$ vs. $\$ 11,889$ USD, respectively).

Another cost-escalator for EVT is the potentially high number of exchange procedures required. The average number of procedures can range from three to five per patient, each of which carries a procedure cost in addition to the intervening cost for in-patient hospitalization. However, when compared with SEMS, the overall duration of hospital stay has been found to be comparable or lower with EVT, and the number of treatment days to be fewer. ${ }^{34}$ With regards to personnel and training, Ward et al. ${ }^{40}$ have estimated that the average time to EVT proficiency for a skilled endoscopist was only ten cases.

\section{FUTURE DIRECTIONS}

Given the established efficacy and safety profile of EVT in the management of esophageal leaks and perforations, 
some groups are now exploring novel avenues for expanding the scope of use. One particularly exciting avenue is the pre-emptive use of EVT at the site of anastomosis following esophagectomy to minimize the risk of leaks (Table 3). ${ }^{3}$ This strategy relies upon the presence of an identifiable feature that predicts the onset of esophageal wall compromise. In this regard, Neumann et al. ${ }^{3}$ explored the emergence of anastomotic ischemia post-esophagectomy as a precursor to the onset of an anastomotic leak to identify patients in whom pre-emptive EVT could promote mucosal recovery. In their case series of eight patients, 75\% (6/8) exhibited complete mucosal recovery after EVT, and 25\% who developed small anastomotic leaks were successfully resolved with ongoing EVT. These early reports helped pave the way for a large multicenter randomized controlled trial developed and led by the team at the University Hospital Zurich known as the 'preSPONGE' trial (NCT04162860). This will explore the efficacy of pre-emptive EVT at the anastomotic site in high-risk patients undergoing minimally invasive esophagectomy postoperatively, including the duration of hospitalization, morbidity, mortality, and 90day anastomotic leak rates.

\section{CONCLUSIONS}

EVT has been extensively demonstrated to be a safe, well-tolerated, effective, versatile, and practicable procedure in the management of selected patients with esophageal wall defects. Furthermore, EVT has demonstrated a superior safety profile compared with alternative treatment strategies. Nevertheless, a number of questions remain regarding the optimal duration of therapy, long-term outcomes, cost-effectiveness, and patient satisfaction. Given the paucity of reported experience globally, we support the implementation of national and international prospective registries of EVT use in esophageal leaks and perforations. Randomized controlled trials, some of which are already underway, will be crucial to further explore the utility, efficacy, and safety of this promising novel technique.

Conflicts of Interest

The authors have no potential conflicts of interest.

Funding

None.

Author Contributions

Conceptualization: Bruno Sgromo, Sotiris Mastoridis

Data curation: Imogen Livingstone, Lily Pollock

Investigation: IL, LP, SM

Project administration: BS, SM
Software: SM

Supervision: BS, SM

Writing-original draft: IL, LP, SM

Writing-review \& editing: IL, LP, BS, SM

ORCID

Imogen Livingstone:

Lily Pollock:

Bruno Sgromo:

Sotiris Mastoridis:

https://orcid.org/0000-0003-0660-5812

https://orcid.org/0000-0002-2818-9488

https://orcid.org/0000-0003-1010-9301

https://orcid.org/0000-0001-7689-2330

\section{REFERENCES}

1. Kuehn F, Schiffmann L, Janisch F, et al. Surgical endoscopic vacuum therapy for defects of the upper gastrointestinal tract. J Gastrointest Surg 2016;20:237-243.

2. Markar SR, Mackenzie H, Wiggins T, et al. Management and outcomes of esophageal perforation: a national study of 2,564 patients in England. Am J Gastroenterol 2015;110:1559-1566.

3. Neumann PA, Mennigen R, Palmes D, Senninger N, Vowinkel T, Laukoetter MG. Pre-emptive endoscopic vacuum therapy for treatment of anastomotic ischemia after esophageal resections. Endoscopy 2017;49:498-503.

4. Dasari BVM, Neely D, Kennedy A, et al. The role of esophageal stents in the management of esophageal anastomotic leaks and benign esophageal perforations. Ann Surg 2014;259:852-860.

5. Wedemeyer J, Schneider A, Manns MP, Jackobs S. Endoscopic vacuum-assisted closure of upper intestinal anastomotic leaks. Gastrointest Endosc 2008;67:708-711

6. Loske G. Endoscopic negative pressure therapy of the upper gastrointestinal tract. Chirurg 2019;90:1-6.

7. Morykwas MJ, Simpson J, Punger K, Argenta A, Kremers L, Argenta J. Vacuum-assisted closure: state of basic research and physiologic foundation. Plast Reconstr Surg 2006;117:121S-126S.

8. Erba P, Ogawa R, Ackermann M, et al. Angiogenesis in wounds treated by microdeformational wound therapy. Ann Surg 2011;253:402-409.

9. Borgquist $\mathrm{O}$, Ingemansson $\mathrm{R}$, Malmsjö $\mathrm{M}$. The influence of low and high pressure levels during negative-pressure wound therapy on wound contraction and fluid evacuation. Plast Reconstr Surg 2011;127:551-559.

10. de Moura DTH, de Moura BFBH, Manfredi MA, et al. Role of endoscopic vacuum therapy in the management of gastrointestinal transmural defects. World J Gastrointest Endosc 2019;11:329-344.

11. Leeds SG, Mencio M, Ontiveros E, Ward MA. Endoluminal vacuum therapy: how I do it. J Gastrointest Surg 2019;23:1037-1043.

12. Ahrens M, Schulte T, Egberts J, et al. Drainage of esophageal leakage using endoscopic vacuum therapy: a prospective pilot study. Endoscopy 2010;42:693-698

13. Loske G, Schorsch T, Müller C. Endoscopic vacuum sponge therapy for esophageal defects. Surg Endosc 2010;24:2531-2535.

14. Wedemeyer J, Brangewitz M, Kubicka S, et al. Management of major postsurgical gastroesophageal intrathoracic leaks with an endoscopic vacuum-assisted closure system. Gastrointest Endosc 2010;71:382-386.

15. Weidenhagen R, Hartl WH, Gruetzner KU, Eichhorn ME, Spelsberg F, Jauch KW. Anastomotic leakage after esophageal resection: new treatment options by endoluminal vacuum therapy. Ann Thorac Surg 2010;90:1674-1681

16. Schorsch T, Müller C, Loske G. Endoscopic vacuum therapy of perforations and anastomotic insufficiency of the esophagus. Chirurg 2014;85:1081-1093

17. Bludau M, Hölscher AH, Herbold T, et al. Management of upper intestinal leaks using an endoscopic vacuum-assisted closure system (E-VAC). Surg Endosc 2014;28:896-901. 
18. Heits N, Stapel L, Reichert B, et al. Endoscopic endoluminal vacuum therapy in esophageal perforation. Ann Thorac Surg 2014;97:1029-1035.

19. Loske G, Schorsch T, Dahm C, Martens E, Müller C. Iatrogenic perforation of esophagus successfully treated with endoscopic vacuum therapy (EVT). Endosc Int Open 2015;3:E547-E551.

20. Laukoetter MG, Mennigen R, Neumann PA, et al. Successful closure of defects in the upper gastrointestinal tract by endoscopic vacuum therapy (EVT): a prospective cohort study. Surg Endosc 2017;31:2687-2696.

21. Bludau M, Fuchs HF, Herbold T, et al. Results of endoscopic vacuum-assisted closure device for treatment of upper GI leaks. Surg Endosc 2018;32:1906-1914.

22. Fraga JC, Nunes DL, Andreolio C, et al. Endoscopic vacuum sponge therapy for an infant with an esophageal leak. J Thorac Cardiovasc Surg 2018;156:e193-e195.

23. Mencio MA, Ontiveros E, Burdick JS, Leeds SG. Use of a novel technique to manage gastrointestinal leaks with endoluminal negative pressure: a single institution experience. Surg Endosc 2018;32:3349-3356.

24. Noh SM, Ahn JY, Lee JH, et al. Endoscopic vacuum-assisted closure therapy in patients with anastomotic leakage after esophagectomy: a single-center experience. Gastroenterol Res Pract 2018;2018:1697968.

25. Ooi G, Burton P, Packiyanathan A, et al. Indications and efficacy of endoscopic vacuum-assisted closure therapy for upper gastrointestinal perforations. ANZ J Surg 2018;88:E257-E263.

26. Pournaras DJ, Hardwick RH, Safranek PM, et al. Endoluminal vacuum therapy (E-Vac): a treatment option in oesophagogastric surgery. World J Surg 2018;42:2507-2511.

27. Still S, Mencio M, Ontiveros E, Burdick J, Leeds SG. Primary and rescue endoluminal vacuum therapy in the management of esophageal perforations and leaks. Ann Thorac Cardiovasc Surg 2018;24:173-179.

28. Cwaliński J, Hermann J, Kasprzyk M, Banasiewicz T. Endoscopic vacuum assisted closure of esophagogastric anastomosis dehiscence: a case report. World J Gastrointest Endosc 2020;12:42-48.

29. Hayami M, Klevebro F, Tsekrekos A, et al. Endoscopic vacuum therapy for anastomotic leak after esophagectomy: a single-center's early experience. Dis Esophagus 2021;34:doaa122.

30. Mastoridis S, Chana P, Singh M, et al. Endoscopic vacuum therapy (EVT) in the management of oesophageal perforations and post-operative leaks. Minim Invasive Ther Allied Technol 2020;1-9.

31. Manfredi MA, Clark SJ, Staffa SJ, et al. Endoscopic esophageal vacuum therapy: a novel therapy for esophageal perforations in pediatric patients. J Pediatr Gastroenterol Nutr 2018;67:706-712.

32. Brangewitz M, Voigtländer T, Helfritz FA, et al. Endoscopic closure of esophageal intrathoracic leaks: stent versus endoscopic vacuum-assisted closure, a retrospective analysis. Endoscopy 2013;45:433-438.

33. Tavares G, Tustumi F, Tristão LS, Bernardo WM. Endoscopic vacuum therapy for anastomotic leak in esophagectomy and total gastrectomy: a systematic review and meta-analysis. Dis Esophagus 2021;34:doaa132.

34. Rausa E, Asti E, Aiolfi A, Bianco F, Bonitta G, Bonavina L. Comparison of endoscopic vacuum therapy versus endoscopic stenting for esophageal leaks: systematic review and meta-analysis. Dis Esophagus 2018;31:doy060.

35. Newton NJ, Sharrock A, Rickard R, Mughal M. Systematic review of the use of endo-luminal topical negative pressure in oesophageal leaks and perforations. Dis Esophagus 2017;30:1-5.

36. Schniewind B, Schafmayer C, Voehrs G, et al. Endoscopic endoluminal vacuum therapy is superior to other regimens in managing anastomotic leakage after esophagectomy: a comparative retrospective study. Surg Endosc 2013;27:3883-3890.

37. Berlth F, Bludau M, Plum PS, et al. Self-expanding metal stents versus endoscopic vacuum therapy in anastomotic leak treatment after oncologic gastroesophageal surgery. J Gastrointest Surg 2019;23:67-75.

38. Heits N, Bernsmeier A, Reichert B, et al. Long-term quality of life after endovac-therapy in anastomotic leakages after esophagectomy. J Thorac Dis 2018;10:228-240.

39. Dhayat SA, Schacht R, Mennigen R, et al. Long-term quality of life assessment after successful endoscopic vacuum therapy of defects in the upper gastrointestinal tract quality of life after EVT. J Gastrointest Surg 2019;23:280-287.

40. Ward MA, Hassan T, Burdick JS, Leeds SG. Endoscopic vacuum assisted wound closure (EVAC) device to treat esophageal and gastric leaks: assessing time to proficiency and cost. Surg Endosc 2019;33:3970-3975. 